\title{
Nominal Exchange Rate Determinacy under the Threat of Currency Counterfeiting
}

\section{By Pedro Gomis-Porqueras, Timothy Kam, and Christopher Wallek*}

\begin{abstract}
We study the endogenous choice to accept fiat objects as media of exchange and their implications for nominal exchange rate determination. We consider a two-country environment with two currencies that can be used to settle any transactions. However, currencies can be counterfeited at a fixed cost and the decision to counterfeit is private information. This induces equilibrium liquidity constraints on the currencies in circulation. We show that the threat of counterfeiting can pin down the nominal exchange rate even when the currencies are perfect substitutes, thus breaking the famous Kareken-Wallace indeterminacy result. (JEL D82, E42, F31)
\end{abstract}

W

hen agents have unrestricted access to currency markets and are free to use any currency as a means of payment, Kareken and Wallace (1981) showed that the rate of return on the two currencies must be identical for both of them to circulate, e.g., they are perfect substitutes. ${ }^{1}$ However, in this case, the nominal exchange rate between these currencies is indeterminate.

Since Kareken and Wallace (1981), most standard models of international monetary economics have considered various departures from their original frictionless environment to generate determinate nominal exchange rates. These include currencies in the utility function, imposing restrictions on the use of currency for certain transactions, assuming differential transaction costs, or having differential terms of trade depending on the currency that is being used. By considering asymmetric treatment of currencies, the nominal exchange rate is determined as currencies then

\footnotetext{
* Gomis-Porqueras: Department of Economics, Deakin University, Victoria 3125, Australia (e-mail: peregomis@gmail.com); Kam: Research School of Economics, H.W. Arndt Bldg 25a, The Australian National University, ACT 0200, Australia (e-mail: tcy.kam@gmail.com); Waller: Research Division, Federal Reserve Bank of St. Louis, P.O. Box 442, St. Louis, MO 63166 (e-mail: cwaller@stls.frb.org). We would like to thank three anonymous referees for their comments. We also thank Guillaume Rocheteau, Yiting Li, Steve Williamson, Ian King, Bruce Preston, Bob King, Charles Engel, Nicolas Jacquet, Jannet Jiang, Ricardo Reis, Cathy Zhang, Russell Wong, Randy Wright, and the participants of the 2014 Summer Workshop on Money, Banking, Payments, and Finance at the Chicago Fed, the 2013 Midwest Macro Meetings, the 8th Workshop on Macroeconomic Dynamics, the 2nd Victoria University Macro Workshop, and the seminar participants at University of Adelaide, University of Queensland, Purdue University, Federal Reserve Bank of St. Louis, and University of California at Irvine. The views expressed are those of the individual authors and do not necessarily reflect official positions of the Federal Reserve Bank of St. Louis, the Federal Reserve System, or the Board of Governors. Companion material can also be found in our online Appendix.

Go to https://doi.org/10.1257/mac.20150172 to visit the article page for additional materials and author disclosure statement(s) or to comment in the online discussion forum.

${ }^{1}$ When currencies are identical in every respect, they are perfect substitutes. Consequently, the composition of currency portfolios is indeterminate for all agents.
} 
become imperfect substitutes. Our objective is to resolve the nominal exchange rate indeterminacy problem while treating the two currencies symmetrically.

In this paper, we study the endogenous choice to accept different fiat objects as media of exchange, the fundamentals that drive their acceptance, and the implications for their bilateral nominal exchange rate. In particular, agents in this economy have no restrictions on what divisible fiat currency can be used to settle transactions. The key aspect of our approach is that we introduce currency fraud as in $\mathrm{Li}$, Rocheteau, and Weill (2012). More precisely, we allow both fiat currencies to be counterfeited at a fixed cost. Potential sellers of goods cannot distinguish genuine from counterfeit currencies and, because of this private information problem, in equilibrium, they put a limit on how much of each currency they are willing to accept. The limits are chosen such that counterfeiting does not occur in equilibrium. The liquidity constraints are endogenous and depend on the relative inflation rates and the cost of counterfeiting. If one of the constraints is binding, the buyer spends all of that currency and uses some of the other currency to "top off" their desired purchase of goods. When both limits bind, the buyer gives up all of his currency holdings to purchase goods.

The most interesting case we study is when the inflation rates and the costs of counterfeiting are exactly the same for each currency. In this case, the only difference between the two currencies is a non-fundamental attribute, such as the color of the currencies. This corresponds exactly to the conditions underlying the Kareken and Wallace (1981) indeterminacy result. Nevertheless, in this case, we show that if the cost of counterfeiting is sufficiently low such that the limits bind, then the nominal exchange rate is determinate and equal to the ratio of the two money stocks. This is interesting because it is the same equilibrium exchange rate that comes out of a standard two-country cash-in-advance model where domestic (foreign) goods must be purchased with domestic (foreign) currency. However, in our model, agents use both currencies to purchase goods. If the constraints do not bind, then the nominal exchange rate is indeterminate.

While our model closely follows Li, Rocheteau, and Weill (2012) in terms of the counterfeiting problem, it differs in two key respects. First, they study counterfeiting of real assets and thus are able to use a finite horizon model. With fiat currencies an infinite horizon is required, which raises issues about the durability of counterfeit currency, which in Li, Rocheteau, and Weill (2012) is not a concern as the economy ends in the same period that counterfeits are detected. ${ }^{2}$ Moreover, because Li, Rocheteau, and Weill (2012) study real assets, relative price indeterminacy does not occur, whereas this is obviously critical when analyzing fiat currencies.

Finally, allowing for counterfeiting is not standard in international monetary economics. While we have changed the environment by introducing a game of private information, we do so symmetrically such that no currency has a counterfeiting advantage over the other. After we present our main results, we describe how differing counterfeiting costs affect the equilibrium of the model.

\footnotetext{
${ }^{2} \mathrm{Li}$, Rocheteau, and Weill (2012) consider an infinite horizon fiat money model in their original working paper. However, there is only one currency, and they make the same assumptions that we do regarding the durability of counterfeits.
} 
In Section I, we review the literature, and in Section II, we describe the model environment. We define the monetary equilibrium and discuss the implications of exchange rate determinacy in Section III. In Section IV, we present more general results for the cases when inflation rates and counterfeiting costs differ. Concluding remarks are found in Section V. Longer proofs and some generalizations are given in our online Appendix.

\section{Related Literature}

Models in mainstream international monetary economics typically pin down the value of a currency by imposing asymmetric assumptions on what objects may be used as media of exchange. For instance, Stockman (1980) and Lucas (1982), among others, assume that in order to buy a good produced by a particular country, the producing country's currency must be used to pay for goods. That is, in these environments, the demand for a specific fiat currency is solely driven by the demand for goods produced by that particular country. Other researchers have introduced local currency in the utility function as in Obstfeld and Rogoff (1984) and Devereux and Engel (2003) or have assumed differential trading cost advantages through network externalities as in Uribe (1997). Devereux and Shi (2013) study a trading post model under the assumption that there is only bilateral exchange at each trading post. Assumptions of this sort determine which currency is used to pay for goods. Consequently, they yield determinacy in agents' portfolio holdings of any two fiat currencies and therefore determinacy of the nominal exchange rate.

Another strand of the international finance literature studies endogenous currency pricing as in Gopinath, Itskhoki, and Rigobon (2010). In this literature firms choose whether to price their goods in domestic currency or foreign currency. The choice of pricing, in conjunction with sticky prices, has implications for exchange rate movements. On the face of it, this sounds as if the firm is choosing which currency to accept as payment. This is not the case-the choice of currency pricing is not equivalent to the choice of currency payment. To illustrate, a firm could choose to price in dollars rather than euros and post a price of $\$ 1$ for a unit of goods. But this does not say the firm accepts only dollars as payment. The firm may accept $\$ 1$ as payment, accept the foreign currency equivalent at the prevailing exchange rate, or some combination of the two. Clearly, fluctuations in the exchange rate affect the quantity of the foreign currency that is paid but the firm is always receiving the equivalent payment of $\$ 1$. This is exactly what Kareken and Wallace (1981) do-firms price in domestic currency but accept either currency as payment. For example, in some New Keynesian international macroeconomic models, such as Clarida, Galí, and Gertler (2002), firms price in domestic currency. In these models, the response of the nominal exchange to shocks in the economy is well-defined. However, the steady state nominal exchange rate is indeterminate since there are no restrictions on currency payments. ${ }^{3}$ So the choice of currency pricing in and of itself has no implications for nominal exchange rate determinacy.

\footnotetext{
${ }^{3}$ In the international New Keynesian models, the assumption of local currency pricing is often imposed. However, this does not imply payment has to be made in the local currency.
} 
In the early search theoretic models of money, agents are able to choose which currencies to accept and use for payment. This literature shows that multiple currencies can circulate even if one is dominated in rate of return and the nominal exchange rate is determinate (see Matsuyama, Kiyotaki, and Matsui 1993; Shi 1995; Zhou 1997; Wright and Trejos 2001; Waller and Curtis 2003; Craig and Waller 2004; and Camera, Craig, and Waller 2004). In these models, currency exchange can occur in bilateral matches if agents' portfolios are overly weighted towards one currency or the other. In fact, this leads to a distribution of determinate nominal exchange rates. However, these findings are driven solely by the decentralized nature of exchange, since agents never have access to a centralized market to rebalance their portfolios. Once agents have the ability to rebalance their currency holdings, be it by the large family assumption as in Shi (1997), or the periodic centralized market structure as in Lagos and Wright (2005), nominal exchange rates are indeterminate as in Kareken and Wallace (1981). To resolve this problem, Head and Shi (2003) consider an environment where the large household can hold a portfolio of currencies but individual buyers are constrained to hold only one currency. So although the household endogenously chooses a portfolio of currencies, bilateral exchange requires using one currency or the other, but not both simultaneously. Similarly, Liu and Shi (2010) assume that buyers can offer any currency but sellers can accept only one currency. The main contribution of our paper relative to the previous search literature is that we include Walrasian markets with centralized exchange without assuming restrictions on currency exchange or differential pricing protocols-and yet we can obtain nominal exchange rate determinacy, even when the currencies are perfect substitutes.

The work closest in spirit to ours is Nosal and Rocheteau (2011, chapter 10.2) and Zhang (2014). These authors have environments where private agents can freely choose which currency to transact with when trading among themselves. Nosal and Rocheteau (2011) consider a two-country variation of the Lagos and Wright (2005) framework and consider a trading mechanism in decentralized markets whereby a buyer obtains better terms of trade in a country by using the domestic currency rather than the foreign one. Despite the asymmetric treatment of the currencies, there are no unexploited gains from trade in decentralized market (DM) so that the outcome is pairwise Pareto efficient and nominal exchange rates are determined. In contrast to these authors, we consider an alternative trading protocol that does not give an advantage of one currency over the other. Finally, Zhang (2014) considers an open economy search model with multiple competing currencies and governments that require transactions to be made in a local currency. Buyers can always costlessly produce counterfeit currencies while sellers face a recognizability problem, as in Lester, Postlewaite, and Wright (2012). The recognizability problem is only in terms of foreign currencies, thus treating currencies asymmetrically and giving one currency an advantage over the other. In this paper, sellers face a counterfeiting problem for both domestic and foreign currencies.

\section{Model}

Consider a two-country model with each country having a non-tradable and tradable sector. Each country is labeled as either Home or Foreign and has a continuum 
of agents of measure 2. Time is discrete and indexed by $t \in \mathbb{N}:=\{0,1,2, \ldots\}$. All agents discount the future at rate $\beta<1$. For ease of understanding, we will describe the model environment from the perspective of the Home country keeping in mind that there is a symmetric Foreign counterpart.

As in Lagos and Wright (2005), in each country there is a sequential decentralized-then-centralized market (DM-CM) trading structure every period. In the DM, a perishable good is traded between Home agents only. ${ }^{4}$ We refer to this market as the non-tradable sector as in Gomis-Porqueras, Kam, and Lee (2013). Traders in this market are either consumers of the DM good or producers of the DM good. DM types are permanent and both have measure 1 . Consumers receive utility $u(q)$ from consuming $q$ units of the DM good with $u(0)=0, u^{\prime}(q)>0$, and $u^{\prime \prime}(q)<0$. Producers incur the disutility cost $c(q)$ from producing $q$ units of the DM good with $c(0)=0, c^{\prime}(q)>0$, and $c^{\prime \prime}(q) \geq 0$. The first best quantity $q^{*} \in(0, \infty)$ satisfies $u^{\prime}\left(q^{*}\right)=c^{\prime}\left(q^{*}\right)$. Consumers and producers meet pair-wise via random matching and bargain over the terms of trade.

Since all agents are anonymous in the DM, credit is not feasible. Hence, a medium of exchange is essential for trade in the DM. Towards this end, consumers can use either the Home currency and/or Foreign currency to pay for $q$. We label consumers in the DM as "buyers" and producers as "sellers." As will be discussed in more detail below, buyers have the ability to counterfeit each currency before trading in the DM and sellers do not have the ability to distinguish between counterfeit currency and genuine currency as in Li, Rocheteau, and Weill (2012). Throughout the rest of the paper we assume that buyers can commit to the terms of trade prior to the counterfeiting decision. Solving this recognizability problem, characterizing the resulting allocations and explaining how nominal exchanges can be determined are the key contributions of our paper.

In the CM, both Home and Foreign agents can produce and consume a homogenous perishable good that is traded internationally in a perfectly competitive, frictionless market. Home agents get utility $\mathcal{U}(C)$ from consuming $C$ units of the $\mathrm{CM}$ good and incur disutility $N$ from producing $N$ units of the CM good. Since this market is frictionless, agents can consume and produce the CM good and they are not anonymous; currencies in this market are not essential for exchange. Agents can adjust their currency portfolios in the CM by selling goods for cash, buying goods to reduce cash holdings, or trading money claims in a complete international market. In this sense, we refer to the $\mathrm{CM}$ as the tradable sector (see also, Gomis-Porqueras, Kam, and Lee 2013), which corresponds to the tradable sector in many international macroeconomic models. The goods price of domestic currency in the CM is denoted by $\phi=1 / P$, where $P$ is the Home currency price of the CM good. Let $e$ be the current nominal exchange rate which measures the value of one unit of Foreign currency $(f)$ in units of the Home currency. Since there is frictionless trade in the CM,

\footnotetext{
${ }^{4}$ The restriction that only the Home agents trade in the DM is for notational and presentational simplicity. A richer environment where Foreign agents can trade with domestic ones in DM does not change the private information game between buyers and sellers that is at the heart of our results. A formal argument that this is the case is shown in our online Appendix.
} 
the law of one price holds. Thus, we have that $P=e P^{f}$, where $P^{f}$ is the Foreign currency price of the CM good. We can rewrite this as $\phi^{f}=e \phi$.

Finally, in order to make a meaningful distinction between genuine and counterfeit currencies, we follow the approach of Nosal and Wallace (2007) and assume that all counterfeit currencies disintegrate at the end of the DM. 5 This makes it costly for DM-sellers to accept counterfeit currency as payment. Although this is an extreme assumption, it simplifies the analysis greatly.

In the date- $t \mathrm{CM}$, the DM-buyers in each country commit to the terms of trade in anticipation of the following DM trade, and make their counterfeiting decisions. This is done before they enter their own country's DM in date $t+1$. Hereinafter, we denote these sequential markets, respectively, as $\operatorname{CM}(t)$ and $\operatorname{DM}(t+1)$. Counterfeiting is costly, requiring a fixed disutility cost of $\kappa$ to counterfeit the Home currency and a fixed cost $\kappa^{f}$ to counterfeit the Foreign currency. While these costs can be different in general, for now we impose $\kappa=\kappa^{f}$ to eliminate any differences in counterfeiting costs. We will expand on the details of the counterfeiting decision later. ${ }^{6}$

Let $M$ and $M^{f}$ denote the stock of Home and Foreign currency, respectively. We assume that the supply of the fiat currencies grow at a constant rate of $\gamma$ and $\gamma^{f}$, respectively. Lump sum transfers of Home and Foreign currencies are made to the respective country's DM-buyers at the beginning of each CM $\tau_{t}=M_{t+1}-M_{t}=(\gamma-1) \gamma^{t-1} M_{0}$ and $\tau_{t}^{f}=M_{t+1}^{f}-M_{t}^{f}=\left(\gamma^{f}-1\right)\left(\gamma^{f}\right)^{t-1} M_{0}^{f}$, respectively. ${ }^{7}$ The initial stocks $M_{0}$ and $M_{0}^{f}$ are known. The gross real returns on each currency from $\mathrm{CM}(t)$ to $\mathrm{CM}(t+1)$ are given by $R_{t}=\phi_{t+1} / \phi_{t}=P_{t} / P_{t+1}=1 / \Pi_{t}$ and $R_{t}^{f}=1 / \Pi_{t}^{f}$, where $\Pi_{t}$ and $\Pi_{t}^{f}$ are the $C M$ inflation rates at home and abroad.

As with the counterfeiting costs, in general, the growth rates of the two currencies can be different; but for the purposes of obtaining our main result, we will impose $\gamma=\gamma^{f}$. This will ensure in equilibrium that the rates of return on the two currencies are the same.

\section{A. Decentralized Market}

At the beginning of each DM, a seller $s$ is randomly matched with a buyer $b$. The buyer enters with a portfolio $A:=\left(a, a^{f}\right)$ consisting of nonnegative amounts of genuine Home currency, Foreign currency, and counterfeits, where $a=m+z$ is the sum of genuine and counterfeit Home currency, and $a^{f}=m^{f}+z^{f}$ is the sum of genuine and counterfeit Foreign currency. The buyer makes a take-it-or-leave-it (TIOLI) offer $\omega:=\left(q, d, d^{f}\right)$ that specifies the quantity $q$ that a seller must produce

\footnotetext{
${ }^{5}$ Alternatively, we could assume that all counterfeits are detected in the CM, confiscated, and destroyed. Our assumption allows us to avoid a discussion of the detection technology and ways of avoiding it.

${ }^{6}$ We refer the reader to our working paper, Gomis-Porqueras, Kam, and Waller (2015), to see how the nominal exchange rate is determined in a closed economy environment with two currencies.

${ }^{7}$ Observe that no transfers are made to DM-sellers as they would have no use for it in the immediate DM. These DM-sellers would just bring the additional money transfer with zero inflation cost into the CM, since this occurs within the same period. In turn, they can afford to work less in the CM without altering their consumption allocation since they have quasi-linear preferences. Thus, it is without loss to our result that we assume that transfers are made to DM-buyers only. Also, if we were to assume that seigniorage revenues were transferred to agents at the start of the CM, the effect of money supply growth would just wash out in the CM in terms of having any real effects since with quasi-linear preferences, all agents would just work less in the CM.
} 
in DM in exchange for $d$ units of the Home currency (genuine and counterfeit) and $d^{f}$ units of the Foreign currency (genuine and counterfeit). ${ }^{8}$ It follows that $d=m_{d}+z_{d}$ and $d^{f}=m_{d}^{f}+z_{d}^{f}$. Feasibility requires $d \leq a$ and $d^{f} \leq a^{f}$.

The seller cannot recognize whether the buyer is offering genuine fiat currencies or not. Thus, she must assign a belief as to the genuineness of the currencies. The seller chooses the probability of accepting the offer. The seller's problem is

$$
\max _{\pi(\omega)} \pi(\omega)\left\{-c(q)+W^{s}\left(\hat{\eta}(\omega) d, \hat{\eta}^{f}(\omega) d^{f}\right)\right\}+[1-\pi(\omega)] W^{s}(0,0),
$$

where $\hat{\eta}(\omega)$ and $\hat{\eta}^{f}(\omega)$ are the seller's beliefs that the Home and Foreign currencies are genuine, and $W^{s}$ denotes the CM value function for the seller.

Given an offer and counterfeiting strategy $\left(\omega, \eta(\omega), \eta^{f}(\omega)\right)$ determined in a preceding CM, and given the buyer's belief $\hat{\pi}(\omega)$ about a seller's probability of accepting his offer, the induced beginning-of-DM value to a buyer with portfolio $\left(a, a^{f}\right)$ is

$$
\begin{aligned}
V^{b}\left(a, a^{f}\right)= & \sigma \hat{\pi}\left\{u(q)+W^{b}\left[\eta(\omega)(a-d), \eta^{f}(\omega)\left(a^{f}-d^{f}\right)\right]\right\} \\
& +[1-\sigma \hat{\pi}(\omega)] W^{b}\left[\eta(\omega) a, \eta^{f}(\omega) a^{f}\right] .
\end{aligned}
$$

Since DM-sellers have no need for currency in the DM, their asset holdings are zero. Thus, the value function for a DM-seller is given by

$$
\begin{aligned}
V^{s}(0,0)=\max _{\pi(\omega)}\{ & \sigma \pi(\omega)\left[-c(q)+W^{s}\left(\hat{\eta}(\omega) d, \hat{\eta}^{f}(\omega) d^{f}\right)\right] \\
+ & {\left.[1-\sigma \pi(\omega)] W^{s}(0,0)\right\}, }
\end{aligned}
$$

where $\pi(\omega)$ is the probability the seller accepts an offer $\omega$ from a randomly encountered buyer, and $W^{s}(0,0)$ is the payoff from walking away from a trade.

\section{B. Centralized Market}

To simplify exposition, we suppress definitions of strategies and the strategy space and refer the reader to Gomis-Porqueras, Kam, and Waller (2015) for more details. In what follows, we denote period $t-1$ variables with the subscript -1 and so on.

Denote $m$ and $m^{f}$ as the quantities of genuine currency brought into the CM. Likewise, $m_{+1}$ and $m_{+1}^{f}$ are the quantities of genuine currencies acquired in the CM, and $z_{+1}$ and $z_{+1}^{f}$ are the quantities of counterfeits of each currency taken into $t+1$. If the buyer does not counterfeit, then $z_{+1}=z_{+1}^{f}=0$. For convenience, let $\left(a_{+1}, a_{+1}^{f}\right):=\left(m_{+1}+z_{+1}, m_{+1}^{f}+z_{+1}^{f}\right)$. Genuine real money balances of Home

\footnotetext{
${ }^{8}$ In our online Appendix, we also consider an alternative proportional bargaining protocol due to Kalai and Smorodinsky (1975). Our main result is robust to this change of trading protocol.
} 
and Foreign currency are given by $\phi m$ and $\phi e m^{f}$, respectively. The flow budget constraint of a DM-buyer in the current CM is

$$
C+\phi m_{+1}+\phi e m_{+1}^{f}=N+\phi m+\phi e m^{f}+\phi \tau .
$$

The DM-buyer, in the current CM, chooses $\left(C, N, \eta(\omega), \eta^{f}(\omega), a_{+1}, a_{+1}^{f}\right)$ to attain

$$
\begin{gathered}
W^{b}\left(m, m^{f}\right)=\max \left\{\mathcal{U}(C)-N-\kappa(1-\eta(\omega))-\kappa^{f}\left(1-\eta^{f}(\omega)\right)\right. \\
\left.+\beta V^{b}\left(a_{+1}, a_{+1}^{f}\right):(3)\right\} .
\end{gathered}
$$

The seller entering the $\mathrm{CM}$ with $\left(m_{s}, m_{s}^{f}\right)$ quantities of genuine currency faces the following problem:

$$
W^{s}\left(m_{s}, m_{s}^{f}\right)=\max _{C, N}\left\{\mathcal{U}(C)-N+\beta V^{s}(0,0): C=N+\phi m_{s}+\phi e m_{s}^{f}\right\} .
$$

Substituting the budget constraint to eliminate $N$, we have that the $\mathrm{CM}$ value functions are linear in currency holdings brought into the CM. In particular, we have that

$$
\begin{aligned}
& W^{b}\left(m, m^{f}\right)=\phi\left(m+e m^{f}+\tau\right)+W^{b}(0,0), \\
& W^{s}\left(m_{s}, m_{s}^{f}\right)=\phi\left(m_{s}+e m_{s}^{f}\right)+W^{b}(0,0) .
\end{aligned}
$$

It follows that the buyer's and seller's $C M$ consumption satisfies $\mathcal{U}^{\prime}\left(C^{*}\right)=1$. Thus, $C^{*}$ is independent of the portfolio choice. Using (1) with (5), updating one period and rearranging terms, yields

$$
\text { (7) } \begin{aligned}
\max _{\omega, m_{+1}, m_{+1}^{f}, \eta(\omega), \eta^{f}(\omega)}\{ & \mathcal{U}\left(C^{*}\right)-\phi\left(m+e m^{f}+\tau\right)+\beta W^{b}(0,0) \\
& -\left(\frac{\phi}{\phi_{+1}}-\beta\right) \phi_{+1} m_{+1}-\kappa(1-\eta(\omega)) \\
& -\left(\frac{\phi e}{\phi_{+1} e_{+1}}-\beta\right) \phi_{+1} e_{+1} m_{+1}^{f}-\kappa^{f}\left(1-\eta^{f}(\omega)\right) \\
& \left.+\beta \sigma \hat{\pi}_{+1}(\omega)\left(u\left(q_{+1}\right)-\eta(\omega) \phi_{+1} d_{+1}-\eta^{f}(\omega) \phi_{+1} e_{+1} d_{+1}^{f}\right)\right\} .
\end{aligned}
$$

The second line and third line of this equation show the expected holding cost (equivalently inflation cost) of acquiring currencies in $t$ for use in $t+1$. The marginal cost of acquiring a unit of genuine Home currency (in real terms) is $\phi_{-1} / \phi-\beta>0$ and $\phi_{-1} e_{-1} / \phi e-\beta>0$ for a unit of real Foreign currency. The rest of the terms are the expected total fixed cost of counterfeiting both currencies. In the fourth line, we have the expected surplus from DM trade. 
Similarly, for the seller we have that

$$
\begin{aligned}
W^{s}\left(m_{s}, m_{s}^{f}\right) & =\mathcal{U}\left(C^{*}\right)-\phi\left(m_{s}+e m_{s}^{f}\right)+\beta W^{s}(0,0) \\
& +\max _{\pi_{+1}(\omega)}\left\{\beta \sigma \pi_{+1}(\omega)\left[-c\left(q_{+1}\right)+\hat{\eta}(\omega) \phi_{+1} d_{+1}+\hat{\eta}^{f}(\omega) \phi_{+1} e_{+1} d_{+1}^{f}\right]\right\} .
\end{aligned}
$$

\section{Counterfeiting Game}

Since the seller is unable to distinguish between counterfeits and genuine currency, whereas the buyer knows what he holds, we have a private information bargaining game. In the environment we examine, buyers are able to post offers in the $\mathrm{CM}$ and commit to honoring them in the ensuing DM. Based on these posted offers, the buyer first chooses the probability of counterfeiting. Then the buyer decides on her portfolio, $A(\omega)$, and CM consumption and effort, $C$ and $N$. After buyers and sellers are matched in the DM, the seller can choose to trade with the buyer at the posted offer. 9 Below we describe the sequence of events in the counterfeiting game that buyers play with sellers.

(i) A DM-buyer announces a TIOLI offer $\omega:=\left(q_{+1}, d_{+1}, d_{+1}^{f}\right)$ and commits to $\omega$ before making any other decisions in $\operatorname{CM}(t)$.

(ii) The buyer chooses the probability of counterfeiting the fiat currencies, $1-\eta(\omega), 1-\eta^{f}(\omega) \in[0,1]$.

(iii) The buyer then decides the portfolio $A_{+1}(\omega)$ and CM consumption and effort $C$ and $N$.

(iv) The buyer enters $\operatorname{DM}(t+1)$, and Nature randomly matches the buyer with a DM-seller with probability $\sigma$.

(v) The DM-seller trades according to the proposed offer $\omega$ with probability $\pi(\omega) \in[0,1]$.

Solving the Private Information Game.--In order to solve the game, we proceed by backward induction from $\operatorname{DM}(t+1)$ to $\mathrm{CM}(t)$. Again, we focus on the events in the Home country as similar conditions can be derived for the Foreign country.

\footnotetext{
${ }^{9}$ This extensive-form game is the same as the endogenous signaling game in Li, Rocheteau, and Weill (2012), where the reordering-invariant refinement proposed by In and Wright (2016) is used to construct the equilibrium. We thank one of the referees for making this point.
} 
In the last stage of the game, in $\operatorname{DM}(t+1)$, the seller maximizes expected profit by playing a mixed strategy $\pi$, where

$$
\text { (9) } \pi_{+1}(\omega) \in\left\{\underset{\pi_{+1}(\omega) \in[0,1]}{\arg \max } \pi_{+1}(\omega)\left[-c\left(q_{+1}\right)+\phi_{+1}\left(\hat{\eta}(\omega) d_{+1}+\hat{\eta}^{f}(\omega) e_{+1} d_{+1}^{f}\right)\right]\right\} \text {, }
$$

taking as given a buyer's offer $\omega$ and the seller's beliefs about the buyer's counterfeiting probabilities, $\left(\hat{\eta}(\omega), \hat{\eta}^{f}(\omega)\right)$.

In the penultimate stage, in $\mathrm{CM}(t)$, the buyer chooses the counterfeiting lottery $\left(\eta(\omega), \eta^{f}(\omega)\right)$ to solve the following cost-minimization problem

$$
\begin{aligned}
& \left\{-\beta \sigma \hat{\pi}_{+1}(\omega)\left[\eta(\omega) \phi_{+1} d_{+1}+\eta^{f}(\omega) \phi_{+1} e_{+1} d_{+1}^{f}\right]-\kappa(1-\eta(\omega))\right. \\
& \left.\quad-\kappa^{f}\left(1-\eta^{f}(\omega)\right)-\left(\frac{\phi}{\phi_{+1}}-\beta\right) \phi_{+1} m_{+1}-\left(\frac{\phi e}{\phi_{+1} e_{+1}}-\beta\right) \phi_{+1} e_{+1} m_{+1}^{f}\right\}
\end{aligned}
$$

given his earlier commitment $\omega$ and his beliefs about a seller's acceptance probability $\hat{\pi}(\omega)$.

The buyer chooses a TIOLI offer at the beginning of the game to maximize his payoff given the conjecture $\left(\hat{\eta}(\omega), \hat{\eta}^{f}(\omega), \hat{\pi}(\omega)\right)$ of the continuation play. The buyer commits to an optimal offer $\omega:=\left(\hat{q}_{+1}, \hat{d}_{+1}, \hat{d}_{+1}^{f}\right)$ to maximize

$$
\begin{aligned}
& \left\{\beta \sigma \hat{\pi}_{+1}(\omega)\left[u\left(\hat{q}_{+1}\right)-\phi_{+1}\left(\hat{\eta}(\omega) \hat{d}_{+1}+\hat{\eta}^{f}(\omega) e_{+1} \hat{d}_{+1}^{f}\right)\right]-\kappa(1-\hat{\eta}(\omega))\right. \\
& \left.\quad-\kappa^{f}\left(1-\hat{\eta}^{f}(\omega)\right)-\left(\frac{\phi}{\phi_{+1}}-\beta\right) \phi_{+1} m_{+1}-\left(\frac{\phi e}{\phi_{+1} e_{+1}}-\beta\right) \phi_{+1} e_{+1} m_{+1}^{f}\right\} .
\end{aligned}
$$

Equilibrium in the Game.-Having specified the seller's and buyer's respective problems, we can now characterize the resulting equilibrium in the privateinformation bargaining game in each country. We show this characterization for the Home country. ${ }^{10}$ From here on, for ease of notation, we will lag the buyer's and seller's events by one period.

PROPOSITION 1: An equilibrium of the counterfeiting-bargaining game is such that

(i) each seller accepts with probability $\hat{\pi}(\omega)=\pi(\omega)=1$;

(ii) each buyer does not counterfeit: $\left(\hat{\eta}(\omega), \hat{\eta}^{f}(\omega)\right)=\left(\eta(\omega), \eta^{f}(\omega)\right)=(1,1)$; and

\footnotetext{
${ }^{10}$ A symmetric description can be written out for the Foreign country.
} 
(iii) each buyer's TIOLI offer $\omega:=\left(q, d, d^{f}\right)$ attains

(12) $\max _{q, d, d^{f}}\left\{-\left(\frac{\phi_{-1}}{\phi}-\beta\right) \phi m-\left(\frac{\phi_{-1} e_{-1}}{\phi e}-\beta\right) \phi e m^{f}+\beta \sigma\left[u(q)-\phi\left(d+e d^{f}\right)\right]\right\}$

subject to

$$
\begin{aligned}
(\zeta): & \phi\left(d+e d^{f}\right)-c(q) \geq 0 \\
(\nu): & 0 \leq d \\
(\mu): & d \leq m, \\
\left(\nu^{f}\right): & 0 \leq d^{f}, \\
\left(\mu^{f}\right): & d^{f} \leq m^{f}, \\
(\lambda): & \phi d \leq \frac{\kappa}{\phi_{-1} / \phi-\beta(1-\sigma)} \\
\left(\lambda^{f}\right): & \phi e d^{f} \leq \frac{\kappa^{f}}{\phi_{-1} e_{-1} / \phi e-\beta(1-\sigma)}
\end{aligned}
$$

and the equilibrium is unique.

The constraints in the characterization above have been written with their respectively Lagrange multipliers denoted in Greek letters in parentheses. The first five constraints of (12) are self-explanatory. The last two, which are the key equilibrium constraints for our results, have the following intuition. Since the buyer does not counterfeit, we have $z=z^{f}=0$ and $d=m, d^{f}=m^{f}$ for $\phi_{-1} / \phi>\beta$ and $\phi_{-1} e_{-1} / \phi e>\beta$. Furthermore, there is no reason to incur the costs of acquiring genuine currencies and then make an offer that the seller will reject, so $\hat{\pi}(\omega)=1$. If the buyer counterfeits, then the marginal cost of producing a counterfeit is zero, while the marginal cost of acquiring genuine currency is positive. In this case, $m, m^{f}=0$. Similarly, there is no reason to incur the cost of counterfeiting if the offer is rejected by the seller, thus $\hat{\pi}(\omega)=1$.

Slightly abusing notation, let $W^{b}\left[\eta, \eta^{f}\right]$ denote the value to a buyer under the pure strategies $\eta(\omega), \eta^{f}(\omega) \in\{0,1\}$, where $\eta=\eta^{f}=1$ represents "no counterfeiting of currencies." For no counterfeiting to be an optimal strategy, it must be the case $W^{b}[1,1] \geq W^{b}[0,1], W^{b}[1,0], W^{b}[0,0]$. Using the expression for the CM value function of the buyer, $W^{b}[1,1] \geq W^{b}[0,1]$ reduces to

$$
\begin{gathered}
-\left(\frac{\phi_{-1}}{\phi}-\beta\right) \phi d-\left(\frac{\phi_{-1} e_{-1}}{\phi e}-\beta\right) \phi e d^{f}+\beta \sigma\left[u(q)-\phi\left(d+e d^{f}\right)\right] \\
\geq-\kappa-\left(\frac{\phi_{-1} e_{-1}}{\phi e}-\beta\right) \phi e d^{f}+\beta \sigma\left[u(q)-\phi e d^{f}\right],
\end{gathered}
$$


which collapses to the second-to-the-last constraint in (12). A similar exercise for $W^{b}[1,1] \geq W^{b}[1,0]$ yields the last constraint in (12). Finally, it is straightforward to show that if the previous two conditions are satisfied, then $W^{b}[1,1] \geq W^{b}[0,0]$ holds as well. We provide the detailed proof behind this intuition in our online Appendix.

In other words, in order to prevent counterfeiting, the seller must put a limit on how much of each currency he is willing to accept. If he is willing to accept more than this limit, then the cost of acquiring genuine currencies is too high so the buyer will choose to counterfeit. Alternatively, above these limits, the marginal cost to the buyer of increasing $d$ and $d^{f}$ is zero, hence the seller must make the marginal benefit of doing so equal to zero. This is achieved by not accepting an offer $\left(d, d^{f}\right)$ above these limits.

\section{Monetary Equilibrium}

We can now embed the equilibrium characterization of the private information games for the Home and Foreign countries into the monetary equilibrium of the two-country model. Since preferences are quasi-linear, the infinite history of past games between buyers and sellers does not matter for each current period agents' decision problems. This allows us to tractably incorporate the equilibrium characterization of the game previously described into the overall dynamic general monetary setting. Before we do so, we return to describing the Home agents' dynamic decision problems.

\section{A. Home Agents'Recursive Problems}

By using the characterization of the counterfeiting game from Proposition 1, the DM-buyers' dynamic problem, lagged one period, simplifies to

$$
\begin{aligned}
\mathcal{Z}_{-1}+\max _{q, d, d^{f}, m, m^{f}}\{ & -\left(\frac{\phi_{-1}}{\phi}-\beta\right) \phi m \\
& \left.-\left[\left(\frac{\phi_{-1} e_{-1}}{\phi e}-\beta\right) \phi e m^{f}+\beta \sigma\left(u(q)-\phi d-\phi e d^{f}\right)\right]\right\}
\end{aligned}
$$

subject to

$$
\begin{aligned}
(\zeta): & \phi\left(d+e d^{f}\right)-c(q)=0 \\
(\nu): & 0 \leq d \\
\left(\nu^{f}\right): & 0 \leq d^{f} \\
(\mu): & d \leq m \\
\left(\mu^{f}\right): & d^{f} \leq m^{f}
\end{aligned}
$$




$$
\begin{gathered}
(\lambda): \quad \phi d \leq \frac{\kappa}{\phi_{-1} / \phi-\beta(1-\sigma)}, \\
\left(\lambda^{f}\right): \quad \phi e d^{f} \leq \frac{\kappa^{f}}{\phi_{-1} e_{-1} / \phi e-\beta(1-\sigma)},
\end{gathered}
$$

where $\mathcal{Z}_{-1}=\mathcal{U}\left(C^{*}\right)-\phi\left(m_{-1}+e_{-1} m_{-1}^{f}+\tau_{-1}\right)+\beta W^{b}(0,0)$, and the Lagrange multiplier for each constraint is listed in parentheses. As before, the first five constraints are straightforward. However, equilibrium in the private information bargaining game introduces the last two liquidity constraints into the buyer's problem in (13).

The corresponding first-order conditions are given by

$$
\begin{aligned}
& 0=\beta \sigma u^{\prime}(q)-\zeta c^{\prime}(q), \\
& \beta \sigma=\zeta+\nu-\mu-\lambda, \\
& \beta \sigma=\zeta+\nu^{f}-\mu^{f}-\lambda^{f}, \\
& \mu=\frac{\phi_{-1}}{\phi}-\beta, \\
& \mu^{f}=\frac{\phi_{-1} e_{-1}}{\phi e}-\beta, \\
& \zeta \geq 0, \nu \geq 0, \nu^{f} \geq 0, \mu \geq 0, \mu^{f} \geq 0, \lambda \geq 0, \lambda^{f} \geq 0,
\end{aligned}
$$

for every date $t \geq 1$.

Equation (14) corresponds to the first-order condition for DM output, which equates the marginal benefit of consuming and the marginal value of the payment to the seller. Since the buyer makes a TIOLI offer, the payment is equal to the seller's DM production cost. Equations (15) and (16) summarize the optimal choice with respect to the two nominal payments: they equate the value of holding a particular fiat currency from one CM to the next versus trading it in DM. Finally, equations (17) and (18) describe the optimal accumulation of each currency, which of course depends on their implied rate of return.

\section{B. Steady-State Monetary Equilibrium}

We now focus on steady-state monetary equilibria where the nominal exchange rate can grow at a constant rate. In steady state, all real quantities are constant such that $\phi M=\phi_{-1} M_{-1}$ and $e \phi M^{f}=e_{-1} \phi_{-1} M_{-1}^{f}$. It then follows that $\Pi \equiv \frac{\phi_{-1}}{\phi}=\gamma$ $=M / M_{-1}$, and the steady-state Home currency (gross) depreciation/appreciation satisfies

$$
\frac{e}{e_{-1}}=\frac{\gamma}{\gamma^{f}}=\frac{\Pi}{\Pi^{f}}
$$


Since we impose that the two currencies grow at the same rate, we have $\gamma=\gamma^{f}$, thus $e / e_{-1}=1$ so there is no currency appreciation or depreciation. In what follows, we explicitly assume $\Pi>\beta$. We will discuss the case of $\Pi=\beta$ later.

Since we assume $\Pi=\Pi^{f}>\beta$, it is straightforward to show that $d=m$ and $d^{f}=m^{f}$. In short, buyers do not acquire costly currency that they do not intend to use. As a result, we have $\zeta>0, \nu=\nu^{f}=0, \mu=\mu^{f}>0$. What remains to be determined is whether $\lambda=\lambda^{f}=0$ or $\lambda=\lambda^{f}>0$. We also impose $\kappa=\kappa^{f}$ $=\tilde{\kappa}=\tilde{\kappa}^{f}$ so that the counterfeiting costs are the same across currencies and across countries. We can now state the main proposition of our paper.

PROPOSITION 2: If $\lambda=\lambda^{f}>0$, then $e=M / M^{f}$.

The key point of this proposition is that even when both currencies are perfect substitutes, e.g., they have the same rates of return and counterfeiting costs, the nominal exchange rate is determinate when both liquidity constraints bind. Thus, we have broken the nominal exchange rate indeterminacy result of Kareken and Wallace (1981). ${ }^{11}$ The fact that sellers face a game of private information generates an upper bound on how much of each currency they are willing to accept in exchange for goods. The no-counterfeiting restrictions in turn create endogenous liquidity constraints for the DM-buyer. Note that if the cost of counterfeiting is low and/or the cost of holding genuine currency is high, then DM-buyers are more willing to engage in counterfeiting. To offset this, DM-sellers have to be more restrictive in the real quantities of each currency that they will accept. Thus, the buyer knows he must bring in a portfolio of currencies with aggregate value $2 \kappa /[\Pi-$ $\beta(1-\sigma)]$. The liquidity constraints prevent the buyer from freely substituting across currencies to achieve this aggregate value since he can bring in no more than $\kappa /[\Pi-\beta(1-\sigma)]$ of either currency. Hence, the liquidity constraints pin down the composition of the buyer's currency portfolio and thus the nominal exchange rate. 12

It is worth noting that the determinate nominal exchange rate obtained above is the same as what comes out of a symmetric, two-country cash-in-advance (CIA) model as in Stockman (1980) and Lucas (1982). While one may be tempted to say that we have provided a "micro-foundation" for the two-country CIA model, this would be incorrect for two reasons. First, our result holds only for a limited set of parameter values. Second, in the standard CIA model, only one of the currencies is used per transaction (by assumption), whereas here both currencies are used in the same transaction.

\footnotetext{
${ }^{11}$ The main insight of this paper is that when sellers face private information regarding the quality of the fiat money, endogenous liquidity constraints arise, and they can help determine the nominal exchange rate. This equilibrium feature is not unique to buyers' take-it-or-leave-it offers. In the online Appendix, we consider proportional bargaining as an alternative trading protocol. We show that when the two liquidity constraints bind, the nominal exchange rate is also the ratio of the money supplies.

${ }^{12}$ Consider the case where $\kappa=\kappa^{f}$ and $\tilde{\kappa}=\tilde{\kappa}^{f}$ but $\kappa \neq \tilde{\kappa}$. In this case, although the currencies are perfect substitutes within a country's DM, they are not perfect substitutes when comparing DMs. It is straightforward to show in this case that if all liquidity constraints bind, we obtain $e=\kappa M / \tilde{\kappa} M^{f}$. As a result, the relative costs of counterfeiting directly influence the exchange rate even though no counterfeiting occurs in equilibrium.
} 
Another interesting aspect of this equilibrium is that, although there is no counterfeiting in equilibrium, the threat of counterfeiting is sufficient to generate determinacy of the nominal exchange rate. We find this interesting because it suggests counterfeiting may be an important factor affecting the equilibrium allocation and nominal exchange rate determinacy. In this sense, it is similar to Kehoe and Levine (1993) and its related literature in which default affects the equilibrium allocation but is not observed in equilibrium.

In addition, we have the following observation regarding efficiency.

COROLLARY 1: The Friedman rule $\Pi=\beta$ is not sufficient to generate the firstbest outcome.

The proof is straightforward. From the first-order conditions in (14)-(19), observe that if $\Pi=\beta$ but $\lambda>0$, we have

$$
\lambda=\beta \sigma\left[\frac{u^{\prime}(q)}{c^{\prime}(q)}-1\right]>0
$$

which implies $q<q^{*}$ in this equilibrium. Since $q$ solves $c(\bar{q})=\kappa / \beta \sigma$, we simply need restrictions on the size of the parameter values to ensure that $\bar{q}<q^{*}$. The key takeaway from this corollary is that the threat of counterfeiting creates an additional friction on the demand for real balances that the Friedman rule does not necessarily overcome.

Having stated our key proposition on nominal exchange determinacy, we now have the following result.

PROPOSITION 3: If $\lambda=\lambda^{f}=0$, then $e$ is indeterminate.

The crux of the problem is that we cannot pin down the composition of the seller's portfolio since buyers can perfectly switch from one currency to the other to acquire the DM good. Consequently, buyers are indifferent as to the quantities of real balances of Home and Foreign currency they hold. Thus, in the absence of binding liquidity constraints, we get the standard indeterminacy result when the currencies are perfect substitutes. It is easy to show that this equilibrium occurs if it is very costly to counterfeit currencies. The key point of this proposition is that nominal exchange rate determinacy does not hold for all parameter configurations of the model as it does in a CIA model or a money in the utility function model.

\section{General Results on Determinacy}

Our main objective was to show that the nominal exchange rate can be determinate even though the currencies are perfect substitutes, differing only by a non-fundamental attribute such as color. However, in general, we can consider cases where the counterfeiting costs and the inflation rates differ across countries. Doing 
so admits a wide variety of equilibrium outcomes. While we do not delve into the details here, we can state the following proposition. ${ }^{13}$

PROPOSITION 4 (Equilibria and Coexistence): Depending on the relative inflation rates of the two fiat currencies, there are three cases characterizing a steady-state monetary equilibrium.

(i) When currency $f$ dominates in rate of return $\left(\Pi>\Pi^{f}\right)$ and

(a) when neither liquidity constraints bind $\left(\lambda=\lambda^{f}=0\right)$, or when only the liquidity constraint on the dominated fiat currency binds $(\lambda>0$, $\lambda^{f}=0$ ), then a monetary equilibrium exists with the unique outcome where only the low-inflation currency circulates; or,

(b) the liquidity constraint on currency $f$ binds $\left(\lambda^{f}>\lambda=0\right)$, then there exists a monetary equilibrium with a unique outcome where the currencies coexist as media of exchange and the nominal exchange rate is determinate:

$$
e=\frac{M}{M^{f}} \frac{\kappa^{f}}{c(q)\left[\Pi^{f}-\beta(1-\sigma)\right]-\kappa^{f}},
$$

where $q$ solves

$$
\frac{\Pi-\beta}{\sigma \beta}=\frac{u^{\prime}(q)-c^{\prime}(q)}{c^{\prime}(q)} .
$$

(c) both liquidity constraints bind $\left(\lambda^{f}>0, \lambda>0\right)$, then there exists a unique monetary equilibrium where the currencies coexist and the nominal exchange rate is determinate:

$$
e=\frac{\kappa^{f} M}{\kappa M^{f}} \frac{\Pi-\beta(1-\sigma)}{\Pi^{f}-\beta(1-\sigma)} .
$$

(ii) When currency $f$ is dominated in rate of return $\left(\Pi^{f}>\Pi\right)$, the coexistence results are the symmetric opposite to those of Case 1.

The intuition for the results follows directly from the special case we studied in the previous propositions. In $i(b)$ and $i(c)$, the exchange rate depreciates according to $(20)$. These results show, as in standard international monetary models, that the higher the Home country's inflation rate is, the faster its currency depreciates. We

\footnotetext{
${ }^{13}$ The proof of this result can be found in Gomis-Porqueras, Kam, and Waller (2015). A proof for a more general setting, where agents can also trade internationally in the DMs, can be found in our online Appendix. For further analysis of the model parameter space where one obtains determinate or indeterminate nominal exchange rate, we also refer the reader to Gomis-Porqueras, Kam, and Waller (2015).
} 
also see that if it is relatively harder to counterfeit the Home currency, then it will be more valuable relative to the Foreign currency.

An interesting feature of $i(b)$ is that even if the Home currency is dominated in rate of return, it still circulates as a medium of exchange. In this equilibrium, it is costly to counterfeit the Home currency ( $\kappa$ is large) but not the Foreign currency ( $\kappa^{f}$ is small). As a result, even though it has a better rate of return, sellers restrict the quantity of the Foreign currency they are willing to accept because they are concerned about counterfeiting. In equilibrium, sellers accept the Foreign currency first but only up to a point, and further purchases of goods are done with the Home currency. This describes countries that are "dollarized"- the Home currency has a worse rate of return, yet the Home currency is not driven out of circulation by the stronger Foreign currency.

Although we have taken $\kappa$ and $\kappa^{f}$ to be exogenous, one could interpret them as being responsive to policy actions. If the Home country undertakes policies to make its currency harder to counterfeit, then equilibrium $i(c)$ above suggests that this will cause the Home currency to appreciate in value (a fall in $e$ ). Since it is harder to counterfeit the Home currency, sellers are willing to accept a larger quantity of the Home currency. This in turn raises the demand for Home currency relative to the Foreign currency.

\section{Conclusion}

In this paper we presented a search theoretic model of two fiat currencies to study the properties of nominal exchange rates when agents face private information. Agents have no restrictions on what divisible fiat currency can be used to settle transactions. Buyers may counterfeit both fiat currencies at a fixed cost while sellers cannot distinguish between counterfeit and genuine fiat currencies. This informational problem gives rise to endogenous liquidity constraints that specify a seller's upper bound on how much fiat currency they are willing to accept. These liquidity constraints have the property that the marginal liquidity value of an additional unit of currency beyond the endogenous binding liquidity constraint is zero. The binding nature of these liquidity constraints is key in determining nominal exchange rates.

When endogenous liquidity constraints on both currencies are binding and the currencies are identical in every respect, we obtain the surprising result that the nominal exchange rate is the ratio of the two money stocks, thus breaking the nominal exchange rate indeterminacy of Kareken and Wallace (1981). When the Foreign currency has a higher rate of return but a lower counterfeiting cost, then the buyer will first pay with the Foreign currency up to the bound and use domestic currency to pay for the remainder of the goods purchased. Because of this, both currencies can circulate even though one currency is dominated in its rate of return. Also, because of this, we can have equilibrium cases in which only one currency emerges as an international currency, while the other currency circulates only locally. The private information problem explored in this paper allows us to break the Kareken and Wallace (1981) indeterminacy result and provides a rationalization of why currencies with dominated rates of return remain in circulation (apart from obvious explanations in terms of legal restrictions) as media of exchange. 


\section{REFERENCES}

Camera, Gabriele, Ben Craig, and Christopher J. Waller. 2004. "Currency competition in a fundamental model of money." Journal of International Economics 64 (2): 521-44.

Clarida, Richard, Jordi Galí, and Mark Gertler. 2002. "A simple framework for international monetary policy analysis.” Journal of Monetary Economics 49 (5): 879-904.

-Craig, Ben, and Christopher J. Waller. 2004. "Dollarization and currency exchange." Journal of Monetary Economics 51 (4): 671-89.

Devereux, Michael B., and Charles Engel. 2003. "Monetary Policy in the Open Economy Revisited: Price Setting and Exchange-Rate Flexibility." Review of Economic Studies 70 (4): 765-83.

-Devereux, Michael B., and Shouyong Shi. 2013. "Vehicle Currency." International Economic Review 54 (1): 97-133.

Gomis-Porqueras, Pedro, Timothy Kam, and Christopher Waller. 2015. "Breaking the Curse of Kareken and Wallace with Private Information." Federal Reserve Bank of St. Louis Research Division Working Paper 2015-028A.

-Gomis-Porqueras, Pere, Timothy Kam, and Junsang Lee. 2013. "Money, Capital, and Exchange Rate Fluctuations." International Economic Review 54 (1): 329-53.

Gopinath, Gita, Oleg Itskhoki, and Roberto Rigobon. 2010. "Currency Choice and Exchange Rate Pass-Through.” American Economic Review 100 (1): 304-36.

Head, Allen, and Shouyong Shi. 2003. "A fundamental theory of exchange rates and direct currency trades." Journal of Monetary Economics 50 (7): 1555-91.

In, Younghwan, and Julian Wright. 2016. "Signaling Private Choices." http://profile.nus.edu.sg/fass/ ecsjkdw/spc160616.pdf.

Kalai, Ehud, and Meir Smorodinsky. 1975. "Other Solutions to Nash's Bargaining Problem.” Econometrica 43 (3): 513-18.

Kareken, John, and Neil Wallace. 1981. "On the Indeterminacy of Equilibrium Exchange Rates." Quarterly Journal of Economics 96 (2): 207-22.

Kehoe, Timothy J., and David K. Levine. 1993. "Debt-Constrained Asset Markets." Review of Economic Studies 60 (4): 865-88.

Lagos, Ricardo, and Randall Wright. 2005. "A Unified Framework for Monetary Theory and Policy Analysis." Journal of Political Economy 113 (3): 463-84.

Lester, Benjamin, Andrew Postlewaite, and Randall Wright. 2012. "Information, Liquidity, Asset Prices, and Monetary Policy." Review of Economic Studies 79 (3): 1209-38.

Li, Yiting, Guillaume Rocheteau, and Pierre-Olivier Weill. 2012. "Liquidity and the Threat of Fraudulent Assets." Journal of Political Economy 120 (5): 815-46.

-Liu, Qing, and Shouyong Shi. 2010. “Currency Areas and Monetary Coordination.” International Economic Review 51 (3): 813-36.

-Lucas, Robert E., Jr. 1982. "Interest rates and currency prices in a two-country world." Journal of Monetary Economics 10 (3): 335-59.

Matsuyama, Kiminori, Nobuhiro Kiyotaki, and Akihiko Matsui. 1993. "Toward a Theory of International Currency." Review of Economic Studies 60 (2): 283-307.

Nosal, Ed, and Guillaume Rocheteau. 2011. Money, Payments, and Liquidity. Cambridge: MIT Press.

- Nosal, Ed, and Neil Wallace. 2007. "A model of (the threat of) counterfeiting." Journal of Monetary Economics 54 (4): 994-1001.

Dobstfeld, Maurice, and Kenneth Rogoff. 1984. "Exchange Rate Dynamics with Sluggish Prices under Alternative Price-Adjustment Rules.” International Economic Review 25 (1): 159-74.

-Shi, Shouyong. 1995. "Money and Prices: A Model of Search and Bargaining." Journal of Economic Theory 67 (2): 467-96.

-Shi, Shouyong. 1997. "A Divisible Search Model of Fiat Money." Econometrica 65 (1): 75-102.

Stockman, Alan C. 1980. "A Theory of Exchange Rate Determination.” Journal of Political Economy 88 (4): 673-98.

-Uribe, Martín. 1997. "Hysteresis in a simple model of currency substitution.” Journal of Monetary Economics 40 (1): 185-202.

-Waller, Christopher J., and Elisabeth S. Curtis. 2003. "Currency restrictions, government transaction policies and currency exchange.” Economic Theory 21 (1): 19-42.

Wright, Randall, and Alberto Trejos. 2001. "International Currency." B. E. Journal of Macroeconomics 1 (1): Article 4.

Zhang, Cathy. 2014. "An information-based theory of international currency." Journal of International Economics 93 (2): 286-301.

Zhou, Ruilin. 1997. "Currency Exchange in a Random Search Model." Review of Economic Studies 64 (2): 289-310. 